\title{
Partial Zero-Forcing Precoding for the Interference Channel with Partially Cooperating Transmitters
}

\author{
Siddarth Hari and Wei Yu \\ Department of Electrical and Computer Engineering \\ University of Toronto, Toronto, ON, Canada \\ Email: \{shari,weiyu\}@ comm.utoronto.ca
}

\begin{abstract}
A communication model is considered in which the classic two-user Gaussian interference channel is augmented by noiseless rate-limited digital conferencing links between the transmitters. We propose a partial zero-forcing precoding strategy based on a shared-private rate splitting scheme at the transmitter, in which each transmitter communicates part of its message to the other transmitter, and subsequently partially pre-subtracts the interfering signal using a zero-forcing precoder. We prove an outer bound and show that the proposed strategy is asymptotically sum-capacity achieving in a very weak interference regime, where both the signal-to-noise ratio (SNR) and the interferenceto-noise ratio (INR) go to infinity while their ratio in $\mathrm{dB}$ scale is kept fixed. In this case, every cooperation bit results in one-bit gain in sum capacity. We also consider a different asymptotic regime where the transmit power constraints and the channel gains are fixed while the noise powers go down to zero. In this case, if one compares with the achievable sum rate with interference treated as noise, one cooperation bit can in fact result in more than one-bit gain in achievable sum rate.
\end{abstract}

\section{INTRODUCTION}

Interference is often the primary limiting factor when multiple uncoordinated links share a common communication medium. The largest known achievable rate region for the classic two-user interference channel is due to Han and Kobayashi [1], where a common-private rate splitting technique is employed to enable the receiver to partially decode and subtract the interfering signal. The Han-Kobayashi scheme has recently been shown to be within one bit of the capacity region of the Gaussian interference channel [2].

This paper studies a communication model in which the classic two-user Gaussian interference channel is augmented by noiseless rate-limited digital conferencing links between the transmitters and explores interference mitigation strategies based on transmitter cooperation. The channel model studied in this paper is related to a number of well-known problems in the literature. If the capacity of the digital link is sufficiently large for each transmitter to describe its entire message to the other transmitter, the resulting channel is a Gaussian vector broadcast channel [3]. If the digital link is unidirectional with sufficiently large capacity, so that one of the transmitters knows the message of the other completely, we have the cognitive radio channel [4].

This paper focuses on communication models in which the conferencing links are rate limited. In this realm, Maric et al. [5] determined the capacity region in the special case of strong-interference regime, where both receivers can decode all messages with no rate penalty. In the weak-interference regime, Bagheri et al. [6] considered the approximate sum capacity of a symmetric interference channel for the case where the digital link between the transmitters is unidirectional. Both of the above works use the conferencing link to share common messages at the transmitter. The idea is to allow transmitters to cooperatively encode the common messages, thereby leading to an increase in common message rates. Both of these works rely on the coding strategy outlined in [7] for the multipleaccess channel with conferencing encoders.

This paper considers an alternative idea of allowing transmitters to share private messages. The idea is to mitigate interference with transmit precoding rather than receiver-based partial decoding and interference subtraction. Toward this end, we propose a shared-private rate splitting technique, which enables each transmitter to communicate its shared message to the other transmitter, and subsequently to pre-subtract part of the interfering signal using a zero-forcing scheme, thereby leading to an increase in private message rates.

The coding strategy considered in this paper is related to that of [8], [9], where quantizing, dirty-paper coding, beamforming and time-sharing strategies for transmitter cooperation are discussed. The main result of this paper is to show that a simple zero-forcing strategy can be quite effective. In fact, in certain weak-interference, high signal-to-noise ratio (SNR) and high interference-to-noise ratio (INR) regime, each cooperation bit at the transmitter can result in one bit increase in overall sum rate.

\section{SySTEM MODEL}

The Gaussian interference channel with a digital link between the transmitters is modelled as follows (see Fig. 1):

$$
\left\{\begin{array}{l}
Y_{1}=h_{11} X_{1}+h_{21} X_{2}+Z_{1} \\
Y_{2}=h_{12} X_{1}+h_{22} X_{2}+Z_{2}
\end{array}\right.
$$

where $X_{1}$ and $X_{2}$ are the transmit signals with power constraints $P_{1}$ and $P_{2}$ respectively, $h_{i j}$ represents the channel gain from transmitter $i$ to receiver $j$, and $Z_{1}, Z_{2}$ are independent additive white Gaussian noises (AWGN) with power $N$. The noiseless digital links between the transmitters have fixed capacities $C_{12}$ and $C_{21}$ respectively.

This paper considers a general conferencing model between the transmitters that allows $K$ conferencing rounds. In particular, the sources 1 and 2 generate random integers $W_{1} \in$ 


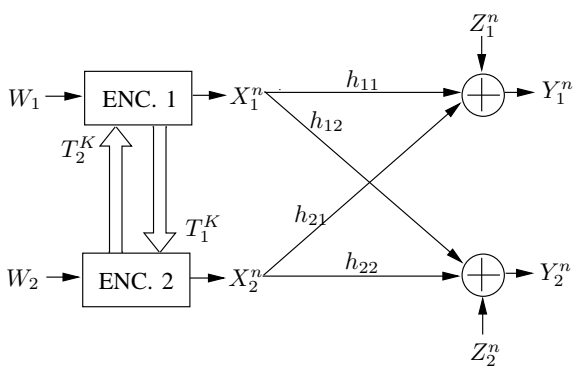

Fig. 1. Interference channel with partially cooperating transmitters

$\left\{1,2, \ldots, 2^{n R_{1}}\right\}$ and $W_{2} \in\left\{1,2, \ldots, 2^{n R_{2}}\right\}$ respectively, at the beginning of each block of $n$ channel uses. Each encoder is completely described by an encoding function and a set of $K$ information sharing functions $\left\{t_{i, 1}, t_{i, 2}, \ldots, t_{i, K}\right\}, i=1,2$. Each function $t_{i, k}$ maps the source message $W_{i}$ and the sequence of previously received communications from the other encoder into the $k$ th communication $T_{i, k}$, where $T_{i, k}$ ranges over a finite alphabet $\vartheta_{i, k}$, for $k=1,2, \ldots, K$. Such a conferencing model was first proposed in [7]. The amount of information that can be exchanged is bounded by the capacities of the digital links between the transmitters:

$$
\sum_{k=1}^{K} \log \left(\left|\vartheta_{i, k}\right|\right) \leq n C_{i j} .
$$

The encoding function maps the source message $W_{i}$ and $T_{j}^{K}=\left(T_{j, 1}, T_{j, 2}, \ldots, T_{j, K}\right)$, the information received from the other transmitter, into a codeword $\mathrm{x}_{\mathbf{i}}=$ $\left(x_{i, 1}, x_{i, 2}, \ldots, x_{i, n}\right)$.

We use the following definitions to simplify the notation:

$$
\begin{array}{ccc}
\mathrm{SNR}_{\mathrm{i}}=\frac{h_{i i}^{2} P_{i}}{N} & \mathrm{INR}_{\mathrm{i}}=\frac{h_{j i}^{2} P_{j}}{N} & \nu_{i}=2^{2 C_{i j}}-1 \\
\gamma(x)=\frac{1}{2} \log (1+x) & \mathrm{h}_{\mathrm{d}}=h_{11} \times h_{22} & \mathrm{~h}_{\mathrm{c}}=h_{12} \times h_{21}
\end{array}
$$

\section{Coding Strategy}

This paper proposes the following cooperation strategy for the interference channel with partially cooperating transmitters. We use only a single conferencing round (i.e., $K=$ 1). Each transmitter splits its source messages $W_{i}$ into a shared $W_{i, s} \in\left\{1,2, \ldots, 2^{n C_{i j}}\right\}$ and a private $W_{i, p} \in$ $\left\{1,2, \ldots, 2^{n\left(R_{i}-C_{i j}\right)}\right\}$ part. We then let each transmitter communicate its shared message to the other transmitter over the digital link. Note that a shared message is only intended to be communicated to the other transmitter-unlike the common message, which is decoded and subtracted at the non-intended receiver.

Since both transmitters know both shared messages, the overall channel can be viewed as a vector broadcast channel with shared messages $\left(U_{1}, U_{2}\right)$, within an interference channel with private messages $V_{1}$ and $V_{2}$. (See Fig. 2). In addition, this paper pursues a simple zero-forcing strategy for the broadcast channel part where the transmitters first encode $\left(U_{1}, U_{2}\right)$ jointly using zero-forcing precoding, while treating $\left(V_{1}, V_{2}\right)$

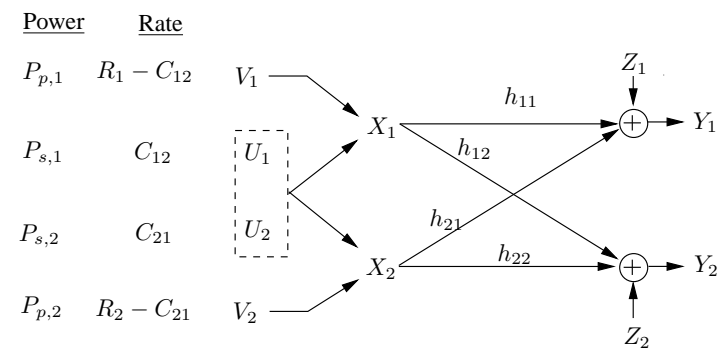

Fig. 2. Transmitter Cooperation via private-shared rate splitting

as interference, then encode $V_{1}$ and $V_{2}$ individually for their respective intended receivers. In effect, the broadcast channel sees the interference channel messages as interference, while the interference channel does not see the broadcast channel messages as interference, because they either are zero-forced at the transmitter or can be decoded-and-subtrcted at the receiver. More specifically, the transmitted signals are

$$
X_{1}=U_{1}-\frac{h_{21}}{h_{11}} U_{2}+V_{1}, \quad X_{2}=U_{2}-\frac{h_{12}}{h_{22}} U_{1}+V_{2}
$$

where $U_{i} \sim \mathcal{N}\left(0, P_{s, i}\right)$ is a codeword representing the shared message $W_{i, s}$ taken from a Gaussian codebook of size $2^{n C_{i j}}$, and $V_{i} \sim \mathcal{N}\left(0, P_{p, i}\right)$ is a codeword representing the private message $W_{i, p}$ taken from a Gaussian codebook of size $2^{n\left(R_{i}-C_{i j}\right)}$.

Because this paper focuses on the zero-forcing strategy only, we constrain the cooperating digital link rates to be below that sufficient to achieve the fully cooperative broadcast channel rate using zero-forcing. More specifically, let $\mathcal{R}^{Z F}$ denote the achievable rate region of the same two-user channel with full transmit cooperation using zero-forcing encoding and under the same per-antenna power constraints. We assume that

$$
\left(C_{12}, C_{21}\right) \in \mathcal{R}^{Z F}
$$

Beyond this region, the entire $\mathcal{R}^{Z F}$ is achievable.

Note that this paper is primarily interested in the high SNR/INR regime, where zero-forcing is near optimal. In the more general case, beamforming and dirty-paper coding can be used for the underlying vector broadcast channel to further improve the achievable rates.

An intuitive understanding of the proposed coding strategy can be obtained by interpreting it in the context of the deterministic interference channel [10]. Fig. 3 illustrates an example where the channel is described by the following parameters: $n_{11}=\left\lfloor\frac{1}{2} \log \left(\mathrm{SNR}_{1}\right)\right\rfloor=3$ bits; $n_{22}=\left\lfloor\frac{1}{2} \log \left(\mathrm{SNR}_{2}\right)\right\rfloor=4$ bits; $n_{21}=\left\lfloor\frac{1}{2} \log \left(\mathrm{INR}_{1}\right)\right\rfloor=2$ bits; $n_{12}=\left\lfloor\frac{1}{2} \log \left(\mathrm{INR}_{2}\right)\right\rfloor=$ 1 bit. When there is no cooperation (Fig. 3(a)), a sum rate of 4 bits is achieved by transmitting only private bits. Fig. 3(b) demonstrates that when $C_{12}=1$ bit, $C_{21}=2$ bits, the achievable sum rate in the deterministic interference channel increases to 7 bits-each cooperation bit improves the sum rate by one bit. 


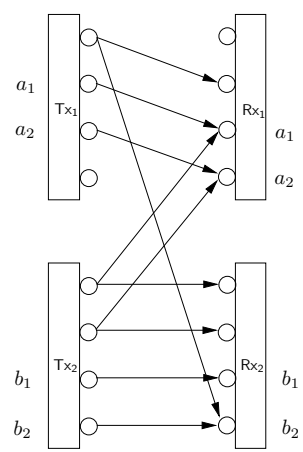

(a)

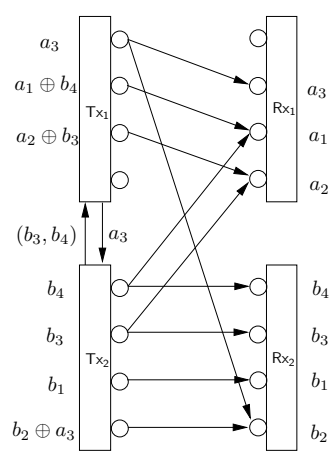

(b)
Fig. 3. Deterministic Interference channel: (a) No cooperation, private message only; (b) $C_{12}=1$ bit, $C_{21}=2$ bits. Transmitter 1 shares bit $a_{3}$. Transmitter 2 shares bits $b_{3}$ and $b_{4}$.

\section{Characterization of CoOperation Gain}

\section{A. Achievable Rate}

The overall achievable rates of the proposed partial zeroforcing scheme can be computed by summing the rates of the respective shared messages and the private messages. The rates of the shared messages are fixed by the capacities of the conferencing links. To compute the private message rates, we first compute the shared and private message powers. The zero-forcing precoding structure of the broadcast channel gives

$$
\left\{\begin{aligned}
& C_{12} \leq \gamma\left(\frac{\left(1-\mathrm{h}_{\mathrm{c}} / \mathrm{h}_{\mathrm{d}}\right)^{2} h_{11}^{2} P_{s, 1}}{N+h_{11}^{2} P_{p, 1}+h_{21}^{2} P_{p, 2}}\right) \\
& C_{21} \leq \gamma\left(\frac{\left(1-\mathrm{h}_{\mathrm{c}} / \mathrm{h}_{\mathrm{d}}\right)^{2} h_{22}^{2} P_{s, 2}}{N+h_{22}^{2} P_{p, 2}+h_{12}^{2} P_{p, 1}}\right) .
\end{aligned}\right.
$$

Further, the power constraints at the transmitters give

$$
\left\{\begin{array}{l}
P_{s, 1}+\left(h_{21}^{2} / h_{11}^{2}\right) P_{s, 2}+P_{p, 1} \leq P_{1} \\
\left(h_{12}^{2} / h_{22}^{2}\right) P_{s, 1}+P_{s, 2}+P_{p, 2} \leq P_{2} .
\end{array}\right.
$$

Setting the inequality constraints as equalities, we have a system of four linear equations in four unknowns, namely $P_{s, 1}, P_{s, 2}, P_{p, 1}$ and $P_{p, 2}$. After some algebra, we obtain the following expressions for the power of the private messages at the transmitters

$$
\begin{array}{r}
P_{p, 1}=\frac{1}{\Delta}\left[\left(\mathrm{h}_{\mathrm{c}}^{2} \nu_{1}+\mathrm{h}_{\mathrm{d}}^{2} \nu_{2}+\left(\mathrm{h}_{\mathrm{d}}-\mathrm{h}_{\mathrm{c}}\right)^{2}\right) P_{1}\right. \\
-\left(\nu_{1}+\nu_{2}\right) h_{21}^{2} h_{22}^{2} P_{2}-N\left(h_{22}^{2} \nu_{1}+h_{21}^{2} \nu_{2}\right) \\
\left.\quad-N \nu_{1} \nu_{2}\left(h_{22}^{2}-h_{21}^{2}\right)\left(\frac{\mathrm{h}_{\mathrm{d}}+\mathrm{h}_{\mathrm{c}}}{\mathrm{h}_{\mathrm{d}}-\mathrm{h}_{\mathrm{c}}}\right)\right]
\end{array}
$$

where

$$
\Delta=\left(\mathrm{h}_{\mathrm{d}}-\mathrm{h}_{\mathrm{c}}\right)^{2}+\left(\mathrm{h}_{\mathrm{d}}^{2}+\mathrm{h}_{\mathrm{c}}^{2}\right)\left(\nu_{2}+\nu_{1}\right)+\left(\mathrm{h}_{\mathrm{d}}+\mathrm{h}_{\mathrm{c}}\right)^{2} \nu_{1} \nu_{2}
$$

and $P_{p, 2}$ is obtained by swapping the indices 1 and 2 in (7). The total achievable rate to receiver $i$ is then given by

$$
R_{i}=\gamma\left(\frac{h_{i i}^{2} P_{p, i}}{N+h_{j i}^{2} P_{p, j}}\right)+C_{i j}
$$

Note that the condition (4) ensures that $P_{p, 1}$ and $P_{p, 2}$ are nonnegative.

\section{B. Low-Noise Regime}

It is instructive to investigate the rate improvement due to the proposed cooperation strategy in the asymptotic low-noise regime with $N \rightarrow 0$ while fixing transmit power and channel gains. Interestingly, if we compare with a baseline case where interference is always treated as noise, the improvement in sum rate due to one bit of transmitter cooperation can be more than one bit.

For an interference channel with no transmit cooperation, if we always treat interference as noise, the asymptotic achievable rates as $N \rightarrow 0$ are

$$
R_{1}=\gamma\left(\frac{\mathrm{SNR}_{1}}{\mathrm{INR}_{1}}\right), \quad R_{2}=\gamma\left(\frac{\mathrm{SNR}_{2}}{\mathrm{INR}_{2}}\right)
$$

Theorem 1: For the interference channel in which the transmitters partially cooperate over digital links of fixed capacities $C_{12}$ and $C_{21}$ satisfying (4), the following rate pair is achievable asymptotically as $N \rightarrow 0$

$$
\begin{aligned}
& R_{1}=\gamma\left(\Gamma \frac{\mathrm{SNR}_{1}}{\mathrm{INR}_{1}}\right)+C_{12} \\
& R_{2}=\gamma\left(\frac{1}{\Gamma} \frac{\mathrm{SNR}_{2}}{\mathrm{INR}_{2}}\right)+C_{21}
\end{aligned}
$$

where

$$
\Gamma=\frac{\mathrm{h}_{\mathrm{c}}^{2} \nu_{1}+\mathrm{h}_{\mathrm{d}}^{2} \nu_{2}+\left(\mathrm{h}_{\mathrm{d}}-\mathrm{h}_{\mathrm{c}}\right)^{2}-\left(\nu_{1}+\nu_{2}\right) h_{21}^{2} h_{22}^{2} P_{2} / P_{1}}{\mathrm{~h}_{\mathrm{d}}^{2} \nu_{1}+\mathrm{h}_{\mathrm{c}}^{2} \nu_{2}+\left(\mathrm{h}_{\mathrm{d}}-\mathrm{h}_{\mathrm{c}}\right)^{2}-\left(\nu_{1}+\nu_{2}\right) h_{12}^{2} h_{11}^{2} P_{1} / P_{2}} .
$$

Proof: Substituting the expressions (7) for $P_{p, 1}$ and $P_{p, 2}$ in (8), and taking the limit as $N \rightarrow 0$, we get (10).

The improvement in sum rate due to rate-limited transmitter cooperation can be more than or less than one-bit-percooperation-bit as compared to (9) depending on the channel parameters. For example, consider channel parameters $h_{11}=$ $1, h_{12}=0.45, h_{21}=0.65, h_{22}=1 ; \mathrm{SNR}_{1}=\mathrm{SNR}_{2}=40 \mathrm{~dB}$, $\mathrm{INR}_{1}=36.3 \mathrm{~dB}, \mathrm{INR}_{2}=33 \mathrm{~dB} ; C_{12}=1$ bit, $C_{21}=2$ bits. The rate pair $(0.875,1.285)$ is achievable treating interference as noise if there is no cooperation between the transmitters. The rate pair $(3.11,2.38)$ is achievable using the coding strategy outlined above. In this case, an exchange of 3 bits between the transmitters results in an improvement of 3.33 bits in the sum rate.

Define

$$
f\left(P_{1}, P_{2}, \mathrm{H}, C_{12}, C_{21}\right)=(\Gamma-1) \frac{\mathrm{SNR}_{1}}{\mathrm{INR}_{1}}+\left(\frac{1}{\Gamma}-1\right) \frac{\mathrm{SNR}_{2}}{\mathrm{INR}_{2}}
$$

we can obtain an asymptotic gain of more than one-bit-perbit if $f>0$. Although $f$ is a messy function of the channel parameters, there are simple cases in which the gain can be easily characterized.

Consider first the symmetric interference channel. Let $\delta$ denote the improvement in sum rate obtained asymptotically 
over (9) as $N \rightarrow 0$, and let $C_{12}=C_{21}=C$. We have

$$
\begin{aligned}
\delta & =\gamma\left(\Gamma \frac{\mathrm{SNR}}{\mathrm{INR}}\right)+\gamma\left(\frac{1}{\Gamma} \frac{\mathrm{SNR}}{\mathrm{INR}}\right)+2 C-2 \gamma\left(\frac{\mathrm{SNR}}{\mathrm{INR}}\right) \\
& =\frac{1}{2} \log \left(\frac{1+\left(\Gamma+\frac{1}{\Gamma}\right) \frac{\mathrm{SNR}}{\mathrm{INR}}+\frac{\mathrm{SNR}}{\mathrm{INR}}^{2}}{1+2 \frac{\mathrm{SNR}}{\mathrm{INR}}+\frac{\mathrm{SNR}}{\mathrm{INR}}^{2}}\right)+2 C \\
& \geq 2 C
\end{aligned}
$$

Thus, $2 C$ cooperation bits result in more than $2 C$ bits improvement in sum rate for the symmetric channel.

The second case is the high signal-to-interference-and-noise (SINR) regime. Here, the sum-rate improvement is

$$
\begin{aligned}
\delta= & \gamma\left(\Gamma \frac{\mathrm{SNR}_{1}}{\mathrm{INR}_{1}}\right)+\gamma\left(\frac{1}{\Gamma} \frac{\mathrm{SNR}_{2}}{\mathrm{INR}_{2}}\right)+C_{12}+C_{21} \\
& -\gamma\left(\frac{\mathrm{SNR}_{1}}{\mathrm{INR}}\right)-\gamma\left(\frac{\mathrm{SNR}_{2}}{\mathrm{INR}_{2}}\right) \\
\approx & \frac{1}{2} \log \left(\Gamma \frac{\mathrm{SNR}_{1}}{\mathrm{INR}_{1}}\right)+\frac{1}{2} \log \left(\frac{1}{\Gamma} \frac{\mathrm{SNR}_{2}}{\mathrm{INR}_{2}}\right)+C_{12}+C_{21} \\
& \quad-\frac{1}{2} \log \left(\frac{\mathrm{SNR}_{1}}{\mathrm{INR}_{1}}\right)-\frac{1}{2} \log \left(\frac{\mathrm{SNR}_{2}}{\mathrm{INR} R_{2}}\right) \\
= & C_{12}+C_{21}
\end{aligned}
$$

Thus, every cooperation bit results in one bit improvement in sum rate in the high SINR regime.

\section{Asymptotic Sum Capacity in the Very Weak INTERFERENCE REGIME}

The main caveat of the results in the previous section is that the rate improvement is computed against a baseline case when interference is treated as noise. Treating interference as noise is not always optimal, particularly in the low-noise regime. As $N \rightarrow 0$, considerable rate gain can be obtained with a Han-Kobayashi strategy that includes common messages.

In this section, instead of letting $N \rightarrow 0$, we investigate a different asymptotic regime, where both SNR and INR go to infinity while their ratio in $\mathrm{dB}$ scale is kept fixed. In a very weak interference regime, we show that every cooperation bit yields exactly one bit improvement in sum rate asymptotically.

\section{A. Sum Capacity Upper Bound}

Theorem 2: The achievable sum rate in the interference channel wherein the transmitters partially cooperate over digital links of fixed capacities $C_{12}$ and $C_{21}$ is bounded by

$$
\begin{aligned}
R_{1}+R_{2} \leq & \min \left\{\mathrm{R}_{\mathrm{bc}}, \gamma\left(\mathrm{INR}_{1}+\frac{\mathrm{SNR}_{1}}{1+\mathrm{INR}_{2}}\right)\right. \\
& \left.+\gamma\left(\mathrm{INR}_{2}+\frac{\mathrm{SNR}_{2}}{1+\mathrm{INR}_{1}}\right)+C_{12}+C_{21}\right\}
\end{aligned}
$$

where $R_{b c}$ is the sum capacity of a Gaussian vector broadcast channel with a two-antenna transmitter and two single-antenna receivers $[3$, Thm. 1$]$.

Proof: Consider a genie-aided channel in which signals $S_{1}=h_{12} X_{1}+Z_{2}$ and $S_{2}=h_{21} X_{2}+Z_{1}$ are available as sideinformation to receivers 1 and 2 respectively. We derive an upper bound for the sum capacity of this genie-aided channel.

Let $\mathrm{C}^{1}$ and $\mathrm{C}^{2}$ be two $\left(2^{n R_{1}}, n\right),\left(2^{n R_{2}}, n\right)$ codes such that $P_{e, 1}^{(n)}, P_{e, 2}^{(n)} \rightarrow 0$, where $P_{e, i}^{(n)}=\operatorname{Pr}\left(\left\{\hat{W}_{i} \neq W_{i}\right\}\right)$. By Fano's inequality, for $n$ sufficiently large

$$
\frac{1}{n} H\left(W_{i} \mid Y_{i}^{n}\right) \leq \frac{1}{n}+R_{i} P_{e, i}^{(n)}<\epsilon .
$$

Since conditioning cannot increase entropy, we have

$$
H\left(W_{i} \mid Y_{i}^{n}, S_{i}^{n}, T_{j}^{K}\right)<n \epsilon
$$

where $S_{i}^{n}$ is the information provided to receiver $i$ by the genie, and $T_{j}^{K}$ is the information provided to transmitter $i$ from transmitter $j$ during the conference. Now,

$$
\begin{aligned}
& n\left(R_{1}+R_{2}\right) \\
& =H\left(W_{1}\right)+H\left(W_{2}\right) \\
& =I\left(W_{1} ; Y_{1}^{n}, S_{1}^{n}, T_{2}^{K}\right)+H\left(W_{1} \mid Y_{1}^{n}, S_{1}^{n}, T_{2}^{K}\right) \\
& +I\left(W_{2} ; Y_{2}^{n}, S_{2}^{n}, T_{1}^{K}\right)+H\left(W_{2} \mid Y_{2}^{n}, S_{2}^{n}, T_{1}^{K}\right) \\
& \stackrel{(a)}{\leq} I\left(W_{1} ; Y_{1}^{n}, S_{1}^{n}, T_{2}^{K}\right)+I\left(W_{2} ; Y_{2}^{n}, S_{2}^{n}, T_{1}^{K}\right)+2 n \epsilon \\
& =I\left(W_{1} ; T_{2}^{K}\right)+I\left(W_{1} ; Y_{1}^{n}, S_{1}^{n} \mid T_{2}^{K}\right) \\
& +I\left(W_{2} ; T_{1}^{K}\right)+I\left(W_{2} ; Y_{2}^{n}, S_{2}^{n} \mid T_{1}^{K}\right)+2 n \epsilon \\
& =H\left(T_{2}^{K}\right)-H\left(T_{2}^{K} \mid W_{1}\right)+I\left(W_{1} ; S_{1}^{n} \mid T_{2}^{K}\right) \\
& +I\left(W_{1} ; Y_{1}^{n} \mid T_{2}^{K}, S_{1}^{n}\right)+H\left(T_{1}^{K}\right)-H\left(T_{1}^{K} \mid W_{2}\right) \\
& +I\left(W_{2} ; S_{2}^{n} \mid T_{1}^{K}\right)+I\left(W_{2} ; Y_{2}^{n} \mid T_{1}^{K}, S_{2}^{n}\right)+2 n \epsilon \\
& \stackrel{(b)}{\leq} H\left(T_{2}^{K}\right)+h\left(S_{1}^{n} \mid T_{2}^{K}\right)-h\left(S_{1}^{n} \mid T_{2}^{K}, W_{1}\right)+h\left(Y_{1}^{n} \mid T_{2}^{K}, S_{1}^{n}\right) \\
& -h\left(Y_{1}^{n} \mid T_{2}^{K}, S_{1}^{n}, W_{1}\right)+H\left(T_{1}^{K}\right)+h\left(S_{2}^{n} \mid T_{1}^{K}\right) \\
& -h\left(S_{2}^{n} \mid T_{1}^{K}, W_{2}\right)+h\left(Y_{2}^{n} \mid T_{1}^{K}, S_{2}^{n}\right) \\
& -h\left(Y_{2}^{n} \mid T_{1}^{K}, S_{2}^{n}, W_{2}\right)+2 n \epsilon \\
& \stackrel{(c)}{\leq} H\left(T_{2}^{K}\right)+h\left(S_{1}^{n} \mid T_{2}^{K}\right)-h\left(S_{1}^{n} \mid X_{1}^{n}\right)+h\left(Y_{1}^{n} \mid T_{2}^{K}, S_{1}^{n}\right) \\
& -h\left(Y_{1}^{n} \mid X_{1}^{n}, S_{1}^{n}\right)+H\left(T_{1}^{K}\right)+h\left(S_{2}^{n} \mid T_{1}^{K}\right)-h\left(S_{2}^{n} \mid X_{2}^{n}\right) \\
& +h\left(Y_{2}^{n} \mid T_{1}^{K}, S_{2}^{n}\right)-h\left(Y_{2}^{n} \mid X_{2}^{n}, S_{2}^{n}\right)+2 n \epsilon \\
& =H\left(T_{2}^{K}\right)+h\left(S_{1}^{n} \mid T_{2}^{K}\right)-h\left(Z_{2}^{n}\right)+h\left(Y_{1}^{n} \mid T_{2}^{K}, S_{1}^{n}\right) \\
& -h\left(S_{2}^{n}\right)+H\left(T_{1}^{K}\right)+h\left(S_{2}^{n} \mid T_{1}^{K}\right)-h\left(Z_{1}^{n}\right) \\
& +h\left(Y_{2}^{n} \mid T_{1}^{K}, S_{2}^{n}\right)-h\left(S_{1}^{n}\right)+2 n \epsilon \\
& \stackrel{(d)}{\leq} h\left(Y_{1}^{n} \mid S_{1}^{n}\right)+h\left(Y_{2}^{n} \mid S_{2}^{n}\right)-h\left(Z_{1}^{n}\right)-h\left(Z_{2}^{n}\right) \\
& +n\left(C_{12}+C_{21}\right)+2 n \epsilon \\
& \stackrel{(e)}{\leq} n\left(\gamma\left(\mathrm{INR}_{1}+\frac{\mathrm{SNR}_{1}}{1+\mathrm{INR}_{2}}\right)\right. \\
& \left.+\gamma\left(\mathrm{INR}_{2}+\frac{\mathrm{SNR}_{2}}{1+\mathrm{INR}_{1}}\right)+C_{12}+C_{21}+2 \epsilon\right)
\end{aligned}
$$

where

(a) follows from Fano's inequality (17);

(b) is due to $H\left(T_{j}^{K} \mid W_{i}\right) \geq 0$;

(c) follows from the fact that the encoding function is a mapping from $\left(W_{i}, T_{j}^{K}\right)$ to $X_{i}^{n}$, so $S_{i}^{n}-X_{i}^{n}-\left(W_{i}, T_{j}^{K}\right)$ forms a Markov chain;

(d) is due to $h\left(S_{i}^{n} \mid T_{j}^{K}\right)-h\left(S_{i}^{n}\right) \leq 0, h\left(Y_{i}^{n} \mid T_{j}^{K}, S_{i}^{n}\right) \leq$ $h\left(Y_{i}^{n} \mid S_{i}^{n}\right)$, and $H\left(T_{i}^{K}\right) \leq n C_{i j}$

(e) follows from [2, Thm. 1]

\section{B. Asymptotic Sum Capacity}

We now characterize the asymptotic sum capacity of a Gaussian interference channel with transmitter cooperation link capacities $C_{12}$ and $C_{21}$ in the limit as $\mathrm{SNR}_{1}, \mathrm{INR}_{1}, \mathrm{SNR}_{2}, \mathrm{INR}_{2} \rightarrow \infty$ while their $\log$ ratios

$$
\alpha_{1}=\frac{\log \left(\mathrm{INR}_{1}\right)}{\log \left(\mathrm{SNR}_{1}\right)} \quad \alpha_{2}=\frac{\log \left(\mathrm{INR}_{2}\right)}{\log \left(\mathrm{SNR}_{2}\right)}
$$


are kept constant. Denote the asymptotic sum capacity as $C_{\text {sum }}^{\infty}\left(C_{12}, C_{21}\right)$. A fundamental result in [2] is that in the very weak interference regime, defined by $\alpha_{1} \leq \frac{1}{2}$ and $\alpha_{2} \leq \frac{1}{2}$,

$$
C_{\text {sum }}^{\infty}(0,0) \approx \frac{1-\alpha_{1}}{2} \log \left(\mathrm{SNR}_{1}\right)+\frac{1-\alpha_{2}}{2} \log \left(\mathrm{SNR}_{2}\right)
$$

where $f(x) \approx g(x)$ denotes $\lim f(x)-g(x)=0$.

Theorem 3: In the very weak interference regime where $\alpha_{1} \leq \frac{1}{2}$ and $\alpha_{2} \leq \frac{1}{2}$, every transmit cooperation bit improves the asymptotic sum capacity by exactly one bit as $\mathrm{SNR}_{1}, \mathrm{INR}_{1}, \mathrm{SNR}_{2}, \mathrm{INR}_{2} \rightarrow \infty$ while $\alpha_{1}$ and $\alpha_{2}$ are kept fixed. More precisely, assuming $C_{12}, C_{21}$ satisfy (4), then

$$
C_{\text {sum }}^{\infty}\left(C_{12}, C_{21}\right)=C_{\text {sum }}^{\infty}(0,0)+C_{12}+C_{21} .
$$

Proof: The achievability follows from the achievable rate computation in Section IV. From (7), we have

$$
\begin{aligned}
& \frac{h_{11}^{2} P_{p, 1}}{N}=\frac{1}{\Delta}\left[\left(\mathrm{h}_{\mathrm{c}}^{2} \nu_{1}+\mathrm{h}_{\mathrm{d}}^{2} \nu_{2}+\left(\mathrm{h}_{\mathrm{d}}-\mathrm{h}_{\mathrm{c}}\right)^{2}\right) \mathrm{SNR}_{1}\right. \\
& \begin{array}{c}
\frac{\left.-\left(\nu_{1}+\nu_{2}\right) \mathrm{h}_{\mathrm{d}}^{2} \mathrm{INR}_{1}+\lambda_{1}\right]}{N}=\frac{1}{\Delta}\left[\left(\mathrm{h}_{\mathrm{c}}^{2} \nu_{2}+\mathrm{h}_{\mathrm{d}}^{2} \nu_{1}+\left(\mathrm{h}_{\mathrm{d}}-\mathrm{h}_{\mathrm{c}}\right)^{2}\right) \mathrm{INR}_{1}\right. \\
\left.-\left(\nu_{1}+\nu_{2}\right) \mathrm{h}_{\mathrm{c}}^{2} \mathrm{SNR}_{1}+\lambda_{2}\right]
\end{array}
\end{aligned}
$$

where $\lambda_{1}, \lambda_{2}$ and $\Delta$ are terms that will eventually vanish in subsequent calculations as $\mathrm{SNR}_{\mathrm{i}}, \mathrm{INR}_{\mathrm{i}} \rightarrow \infty$. Now, since $\alpha_{1}$ and $\alpha_{2}$ are fixed,

$$
\mathrm{INR}_{1}=\mathrm{SNR}_{1}{ }^{\alpha_{1}} \quad \mathrm{INR}_{2}=\mathrm{SNR}_{2}{ }^{\alpha_{2}}
$$

Substituting (19) and (20) in (8), we get

$$
\begin{aligned}
& R_{1}=\gamma\left(\begin{array}{c}
{\left[\mathrm{h}_{\mathrm{c}}^{2} \nu_{1}+\mathrm{h}_{\mathrm{d}}^{2} \nu_{2}+\left(\mathrm{h}_{\mathrm{d}}-\mathrm{h}_{\mathrm{c}}\right)^{2}\right] \mathrm{SNR}_{1}} \\
\frac{-\left(\nu_{1}+\nu_{2}\right) \mathrm{h}_{\mathrm{d}}^{2} \mathrm{SNR}_{1}{ }^{\alpha_{1}}+\lambda_{1}}{\left[\mathrm{~h}_{\mathrm{c}}^{2} \nu_{2}+\mathrm{h}_{\mathrm{d}}^{2} \nu_{1}+\left(\mathrm{h}_{\mathrm{d}}-\mathrm{h}_{\mathrm{c}}\right)^{2}\right] \mathrm{SNR}_{1}{ }^{\alpha_{1}}} \\
-\left(\nu_{1}+\nu_{2}\right) \mathrm{h}_{\mathrm{c}}^{2} \mathrm{SNR}_{1}+\lambda_{2}+\Delta
\end{array}\right)+C_{12} \\
& \approx \gamma\left(\Gamma_{1} \mathrm{SNR}_{1}^{1-\alpha_{1}}\right)+C_{12}, \quad \text { as SNR }_{1} \rightarrow \infty
\end{aligned}
$$

where

$$
\Gamma_{1}=\frac{\mathrm{h}_{\mathrm{c}}^{2} \nu_{1}+\mathrm{h}_{\mathrm{d}}^{2} \nu_{2}+\left(\mathrm{h}_{\mathrm{d}}-\mathrm{h}_{\mathrm{c}}\right)^{2}-\left(\nu_{1}+\nu_{2}\right) \mathrm{h}_{\mathrm{d}}^{2} \mathrm{SNR}_{1}^{\alpha_{1}-1}}{\mathrm{~h}_{\mathrm{c}}^{2} \nu_{2}+\mathrm{h}_{\mathrm{d}}^{2} \nu_{1}+\left(\mathrm{h}_{\mathrm{d}}-\mathrm{h}_{\mathrm{c}}\right)^{2}-\left(\nu_{1}+\nu_{2}\right) \mathrm{h}_{\mathrm{c}}^{2} \mathrm{SNR}_{1}^{1-\alpha_{1}}}
$$

Similarly,

$$
R_{2} \approx \gamma\left(\Gamma_{2} \mathrm{SNR}_{2}^{1-\alpha_{2}}\right)+C_{21}, \quad \text { as } \mathrm{SNR}_{2} \rightarrow \infty
$$

where

$$
\Gamma_{2}=\frac{h_{c}^{2} \nu_{2}+h_{d}^{2} \nu_{1}+\left(h_{d}-h_{c}\right)^{2}-\left(\nu_{1}+\nu_{2}\right) h_{d}^{2} S^{2} R_{2}^{\alpha_{2}-1}}{h_{c}^{2} \nu_{1}+h_{d}^{2} \nu_{2}+\left(h_{d}-h_{c}\right)^{2}-\left(\nu_{1}+\nu_{2}\right) h_{c}^{2} \operatorname{SNR}_{2}^{1-\alpha_{2}}}
$$

It is easily verified that $h_{c}^{2} S N R_{2}^{1-\alpha_{2}}=h_{d}^{2} S N R_{1}^{\alpha_{1}-1}$. It immediately follows that $\Gamma_{1} \Gamma_{2}=1$. Therefore, as $\mathrm{SNR}_{1}, \mathrm{SNR}_{2} \rightarrow \infty$, we have

$$
\begin{aligned}
R_{1} & +R_{2} \approx \gamma\left(\Gamma_{1} \mathrm{SNR}_{1}^{1-\alpha_{1}}\right)+\gamma\left(\Gamma_{2} \mathrm{SNR}_{2}^{1-\alpha_{2}}\right)+C_{12}+C_{21} \\
& \approx \frac{1}{2} \log \left(\Gamma_{1} \mathrm{SNR}_{1}^{1-\alpha_{1}}\right)+\frac{1}{2} \log \left(\Gamma_{2} \mathrm{SNR}_{2}^{1-\alpha_{2}}\right)+C_{12}+C_{21} \\
& =\frac{1-\alpha_{1}}{2} \log \left(\mathrm{SNR}_{1}\right)+\frac{1-\alpha_{2}}{2} \log \left(\mathrm{SNR}_{2}\right)+C_{12}+C_{21}
\end{aligned}
$$

The converse follows from the outer bound in Theorem 2:

$$
\begin{aligned}
& R_{1}+R_{2} \leq \gamma\left(\mathrm{INR}_{1}+\frac{\mathrm{SNR}_{1}}{1+\mathrm{INR}_{2}}\right) \\
&+\gamma\left(\mathrm{INR}_{2}+\frac{\mathrm{SNR}_{2}}{1+\mathrm{INR}_{1}}\right)+C_{12}+C_{21} \\
&<\frac{1}{2} \log \left(\frac{\mathrm{SNR}_{1}}{\mathrm{INR}}\right)+\frac{1}{2} \log \left(\frac{\mathrm{INR}_{1}}{\mathrm{SNR}_{1}}+\frac{\mathrm{INR}_{1}^{2}}{\mathrm{SNR}_{1}}+\frac{\mathrm{INR}_{1}}{\mathrm{INR}_{2}}\right) \\
&+\frac{1}{2} \log \left(\frac{\mathrm{SNR}_{2}}{\mathrm{INR}}\right)+\frac{1}{2} \log \left(\frac{\mathrm{INR}_{2}}{\mathrm{SNR}_{2}}+\frac{\mathrm{INR}_{2}}{\mathrm{SNR}_{2}}+\frac{\mathrm{INR}_{2}}{\mathrm{INR}_{1}}\right) \\
& \quad+C_{12}+C_{21} \\
&=\frac{1-\alpha_{1}}{2} \log \left(\mathrm{SNR}_{1}\right)+\frac{1-\alpha_{2}}{2} \log \left(\mathrm{SNR}_{2}\right)+C_{12}+C_{21} \\
&+\frac{1}{2} \log \left(\mathrm{SNR}_{1}^{\alpha_{1}-1}+\mathrm{SNR}_{1}^{2 \alpha_{1}-1}+\frac{\mathrm{INR}_{1}}{\mathrm{INR}_{2}}\right) \\
&+\frac{1}{2} \log \left(\mathrm{SNR}_{2}^{\alpha_{2}-1}+\mathrm{SNR}_{2}^{2 \alpha_{2}-1}+\frac{\mathrm{INR}_{2}}{\mathrm{INR}_{1}}\right)
\end{aligned}
$$

The sum of the last two terms vanishes as $\mathrm{SNR}_{1}, \mathrm{SNR}_{2} \rightarrow \infty$ for $\alpha_{1}, \alpha_{2}<1 / 2$. This completes the proof.

\section{CONCLUding REMARKS}

This paper explores the use of cooperation link at the transmitters for interference mitigation. We propose a zeroforcing strategy which pre-subtracts part of the interfering signal. This strategy is particularly effective in high SNR/INR regimes-it gives one bit of sum-capacity improvement for every cooperation bit in a very weak interference regime; it can yield more than one-bit-per-cooperation-bit when compared with the case where interference is treated as noise.

\section{REFERENCES}

[1] T. Han and K. Kobayashi, "A new achievable rate region for the interference channel," IEEE Trans. Inf. Theory, vol. 27, no. 1, pp. 49-60, Jan 1981.

[2] R. Etkin, D. Tse, and H. Wang, "Gaussian interference channel capacity to within one bit," IEEE Trans. Inf. Theory, vol. 54, no. 12, pp. 55345562, Dec. 2008.

[3] G. Caire and S. Shamai, "On the achievable throughput of a multiantenna Gaussian broadcast channel," IEEE Trans. Inf. Theory, vol. 49, no. 7, pp. 1691-1706, July 2003.

[4] N. Devroye, P. Mitran, and V. Tarokh, "Achievable rates in cognitive radio channels," IEEE Trans. Inf. Theory, vol. 52, no. 5, pp. 1813-1827, May 2006.

[5] I. Maric, R. Yates, and G. Kramer, "Capacity of interference channels with partial transmitter cooperation," IEEE Trans. Inf. Theory, vol. 53 no. 10, pp. 3536-3548, Oct. 2007.

[6] H. Bagheri, A. Motahari, and A. Khandani, "Generalized degrees of freedom of the symmetric Gaussian interference channel with partial unidirectional transmitter cooperation," in 43rd Conf. Inform. Sci. Sys. (CISS), March 2009, pp. 307-312.

[7] F. Willems, "The discrete memoryless multiple access channel with partially cooperating encoders (corresp.)," IEEE Trans. Inf. Theory, vol. 29, no. 3, pp. 441-445, May 1983.

[8] P. Marsch and G. Fettweis, "On base station cooperation schemes for downlink network MIMO under a constrained backhaul," in IEEE Global Telecommun. Conf. (GLOBECOM), 2008.

[9] — , "On downlink network MIMO under a constrained backhaul and imperfect channel knowledge," in IEEE Global Telecommun. Conf., (GLOBECOM), 2009.

[10] G. Bresler and D. Tse, "The two-user Gaussian interference channel: a deterministic view," European Trans. Telecommun., vol. 19, no. 4, pp. 333-354, 2008. 